\title{
Aspiration versus tube drainage in primary spontaneous pneumothorax: a randomised
} study

\author{
A.K. Ayed*,\#, C. Chandrasekaran ${ }^{\#}$ and M. Sukumar ${ }^{\#}$
}

ABSTRACT: This randomised study was designed to compare clinical outcomes for simple aspiration versus tube thoracostomy, in the treatment of the first primary spontaneous pneumothorax (PSP) attack. A randomised trial, comparing simple aspiration with tube thoracostomy, in 137 patients with a first episode of PSP was carried out.

Immediate success was obtained in 40 out of the 65 patients (62\%) randomly assigned to undergo simple aspiration and in 49 out of the 72 patients $(68 \%)$ who had been randomly assigned to undergo tube thoracostomy. The 1-week success rates were: 58 (89\%) patients in the intentionto-treat simple aspiration group and $63(88 \%)$ patients in the tube thoracostomy group. In the aspiration group, there were more recurrences during the 3-month follow-up period (15 versus $8 \%)$, though the difference was not significant. Recurrence rates at 1 and 2 yrs were $16(22 \%)$ and $20(31 \%)$ for patients who had undergone simple aspiration, respectively, and $17(24 \%)$ and 18 (25\%) for patients who had undergone tube thoracostomies, respectively. Complications occurred in $5(7 \%)$ patients who had undergone a tube thoracostomy and $1(2 \%)$ patient who had undergone simple aspiration. Analgesia was required in 22 (34\%) patients of the simple aspiration group versus 40 (56\%) patients of the tube thoracostomy group.

These findings suggest that simple aspiration could be an acceptable alternative to tube thoracostomy in the treatment of primary spontaneous pneumothorax.

KEYWORDS: Aspiration, recurrence, spontaneous pneumothorax, tube thoracostomy

D rimary spontaneous pneumothorax (PSP) is common in young, tall, thin people without clinically apparent underlying lung disease. The incidence is estimated at 1828 per 100,000 for males and $1.2-6$ per 100,000 for females [1]. Several options are available for the treatment of PSP. These include observation, oxygen supplementation, simple aspiration, tube thoracostomy, video-assisted thoracoscopy surgery (VATS) and thoracotomy [2-4]. The goal of first-attack PSP therapy is to eliminate the intrapleural air collection, thereby allowing reexpansion of the lung. In general, if the pneumothorax is small, i.e. it occupies $<20 \%$ of the hemithorax and the patient is asymptomatic, observation alone is adequate. If the pneumothorax is $>20 \%$, or the patient is symptomatic, evacuation of air from the pleural space, by simple aspiration or tube thoracostomy, is indicated [5]. The choice of method is still controversial. The American College of Chest Physicians released expert-based consensus on the management of PSP using the Delphi method in 2001 [5]. Chest tube or pleural catheters were recommended as the preferred intervention, over simple observation. The level of consensus for this was graded as good. They advocated simple aspiration only for stable patients with small PSP that progressed with observation, but otherwise found it to be an inappropriate treatment option [5]. However, the British Thoracic Society guidelines recommend that simple aspiration should be the treatment of choice for stable PSP, which requires intervention, regardless of the size of pneumothorax $[6,7]$. To date, only one randomised, prospective, multicentre pilot study, comparing manual aspiration to chest tube in patients with a first attack of PSP has been published [8]. Other randomised studies had mixed patient populations (primary and secondary
AFFILIATIONS

*Dept of Surgery, Faculty of Medicine, Kuwait University and \#Dept of Thoracic Surgery, Chest Diseases Hospital, Safat, Kuwait.

CORRESPONDENCE

A.K. Ayed

Kuwait University

Faculty of Medicine

Dept of Surgery

P.0. Box 24923

Safat 13110

Kuwait

Fax: 9655319579

E-mail: Adel@hsc.edu.kw

Received:

August 052005

Accepted after revision:

November 172005 
pneumothorax, first and recurrent attacks PSP) $[9,10]$. The present study was designed to evaluate the efficacy and complications of aspiration versus tube thoracostomy for patients with a first episode of PSP and patients were randomly assigned to one of two therapeutic methods.

\section{METHODS}

\section{Patients}

The study was conducted in the Chest Diseases Hospital (Safat, Kuwait), which is the only centre for the treatment of chest disorders in Kuwait. Patients were randomly assigned to a procedure if the member of the team was in agreement that the patient with a documented first attack PSP was considered eligible for inclusion, that they were symptomatic (dyspnoea and chest pain) regardless of the size of the pneumothorax or if the pneumothorax size was $>20 \%$ of the hemithorax. Exclusion criteria included: the history of previous pneumothorax, secondary spontaneous pneumothorax, tension pneumothorax, bilateral pneumothorax, iatrogenic pneumothorax, asymptomatic $<20 \%$ PSP and hemipneumothorax. The institutional Ethics Review Committee approved this study with both verbal and written informed consent having been obtained from all participants, prior to enrolment and randomisation. After confirmation of absence of exclusion criteria, 137 consecutive patients from January 2001 to January 2003 were included. None of the patients refused to participate in the trial. Randomisation was performed by self-selecting a sealed envelop that indicated the respective treatment.

\section{Simple aspiration}

Simple aspiration was performed whilst the patients were lying in a semi-supine position. After the skin had been prepared and a local anaesthetic had been administered, a plastic catheter (size 16 Gauge: Argyle, Sherwood Medical, Tullamore, Ireland) was inserted into the second anterior intercostal space on the midclavicular line. The catheter was connected to a one-bottle water seal vacuum system, regulated to generate a negative pressure of $10-15 \mathrm{cmH}_{2} \mathrm{O}$. Aspiration was performed until cessation of air occurred in the water seal bottle or for a maximum of 30 mins. Thereafter, the catheter was withdrawn and a chest radiograph was performed. If the lung expansion was complete, or if only a small rim of apical pneumothorax was present, the patient was discharged. If no lung expansion or only partial expansion was obtained, a second aspiration attempt through a newly inserted catheter, at the same skin site, was performed immediately. If a second attempt was successful the patient was discharged. If a second attempt was unsuccessful or a continuous air leak was observed, at the one-bottle water seal vacuum system, a tube thoracostomy was performed. After discharge, patients were seen at 1 week, 3, 6, 12 and 24 months, or when indicated.

\section{Tube thoracostomy}

A tube thoracostomy was performed with 20F plastic tubes (Argyle, Sherwood Medical). The chest tube was inserted under local anaesthesia, at the fourth or fifth interspace at the midaxillary line, and directed towards the apex. The drain was connected to an underwater seal suction with a negative pressure of $20 \mathrm{cmH}_{2} \mathrm{O}$. When the air had stopped bubbling and a chest radiograph had confirmed lung expansion, the drain was left connected to the water seal for a further $24 \mathrm{~h}$. After a control chest radiograph, the drain was removed and the patient was allowed home. Chest tube drainage was prolonged if the air leak persisted and incomplete lung expansion remained after 7 days. Thereafter, subsequent treatment (VATS) was performed. After discharge, patients were seen at 1 week, 3, 6, 12 and 24 months, or earlier if indicated. Any recurrence was proved by a chest radiograph during a followup visit. Pethidine, an intra muscular (IM) analgesic, was administered every $4-6 \mathrm{~h}$, according to the patient's request, and oral analgesia (acetaminophen) was administered as needed.

\section{End points}

The true primary end point of the trial was immediate success. Immediate success for simple aspiration was defined as complete, or nearly complete, lung expansion following aspiration. Immediate success for a tube thoracostomy was defined as absence of air leak, complete lung expansion and chest tube removal within 3 days of the insertion of the tube.

The secondary end points included 1-week success, recurrence rate at 3 months, 1-yr and 2-yr follow-up for both groups, hospitalisation, hospital stay, analgesia requirements and quantity of analgesia in $24 \mathrm{~h}$, complications and inability to work. A 1-week success rate was defined on an intention-totreat basis in both groups. In the simple aspiration group, a subsequent tube thoracostomy was proposed in cases of immediate failure. A 1-week success of simple aspiration on an intention-to-treat basis was, therefore, defined as complete lung expansion at 7 days post second attempt of aspiration (followed by a tube thoracostomy in case of unsuccessful aspiration). In the tube thoracostomy group, a 1-week success was defined as complete lung expansion followed by tube removal within 7 days post tube insertion. Success was defined as no recurrence at each follow-up visit.

\section{Statistical analysis}

Data were expressed as mean \pm SD. The cut-off level for statistical significance was $\mathrm{p}<0.05$. The unpaired t-test was used to assess the significance between means of variables in the groups. The Pearson's Chi-squared test was used to ascertain the significance of association between two categorical variables. The Chi-squared test was replaced by Fisher's exact test if the cell frequencies of any of the $2 \times 2$ contigency tables $<5$. The sample size is believed to guarantee precision in detecting significance between the two groups at type I error 0.05 and power $80 \%$. For difference in proportions, Fisher's exact test was used to generate p-values and Woolf's approximation was used to calculate $95 \%$ confidence intervals around the difference.

\section{RESULTS}

Between January 2001 and January 2003, 208 patients were enrolled in the study and 137 patients underwent randomisation. Of the 71 patients who were not randomly assigned to treatment groups, 42 were recurrent PSP patients and offered VATS, 16 with asymptomatic small PSP and were treated by observation, 10 had a tension pneumothorax, and three had a haemopneumothorax. The baseline patient clinical characteristics for each group are shown in table 1 . There were no differences in age, sex, weight, body mass index, smoking status or radiographical appearances. 


\begin{tabular}{lccc}
\hline TABLE 1 & $\begin{array}{c}\text { Characteristics of patients randomly assigned to } \\
\text { treatment groups }\end{array}$ \\
\hline Parameters & Aspiration & Tube thoracostomy & p-value \\
\hline Sex $\mathbf{n}$ & & & 0.2 \\
$\quad$ Male & 59 & 69 & \\
Female & 6 & 3 & \\
Age yrs & $24.38 \pm 4.4$ & $23.5 \pm 4.8$ & 0.3 \\
Weight kg & $56.1 \pm 10.7$ & $57.1 \pm 11.7$ & 0.6 \\
BMI & $19.1 \pm 2.8$ & $19.8 \pm 3.6$ & 0.5 \\
Smokers $\mathbf{n}$ & & & 0.5 \\
Yes & 53 & 55 & \\
$\quad$ No & 12 & 17 & \\
Radiographical & & & \\
appearance & & & \\
(PTX) $\mathbf{n}$ & & 30 & \\
Left & 20 & 42 & 0.1 \\
Right & 45 & 54 & \\
Partial & 51 & 18 & \\
Complete & 14 & & \\
\hline
\end{tabular}

Data are presented as mean \pm SD unless otherwise stated. BMI: body mass index; PTX: pneumothorax.

Study end points analyses for both groups are shown in table 2 and figure 1 . Immediate success rates were similar in both groups; $40(62 \%)$ and $49(68 \%)$ patients for simple aspiration and tube thoracostomy, respectively.

Of the 25 patients in whom simple aspiration was unsuccessful at first attempt, a second attempt was successful in 13. In the remaining 12 patients, tube thoracostomy was performed, which was successful within a week for five of the patients. Seven required VATS due to the presence of a persistent air leak after 1 week. Therefore, in the intention-to-treat simple aspiration group, 58 (89\%) were successful for simple aspiration followed by tube thoracostomy within 1 week. None of the patients after discharge, following simple aspiration, had to be readmitted for recurrence or complications. The earliest recurrence occurred 4 weeks after discharge.

In the tube thoracostomy group, treatment was successful within 3 days in 49 patients (68\%). In 14 of the remaining 23 patients, prolonged chest drainage was successful after 1 week, while in nine other patients VATS was needed for a persistent air leak. Therefore, the 1 -week success rate for tube thoracostomy was 63 patients $(88 \%)$.

In the simple aspiration group, 17 patients (26\%) were admitted to hospital, with a mean stay of $1.8 \pm 3.9$ days (range: 1-13 days). They included the 17 patients in whom tube thoracostomies were performed. All patients in the tube thoracostomy group were admitted, with a mean stay of $4.04 \pm 2.9$ days (range: 2-13 days). The earliest recurrence occurred 7 weeks after discharge.

Recurrence rate at the 3-month follow-up check was 15\% (10 out of 65 patients) in simple aspiration group and $8 \%$ (six out of 72 patients) in the tube thoracostomy group $(p=0.2)$. Recurrence rate at 1- and 2-yr follow-up were similar in both groups. Recurrences were treated with VATS in all patients. The mean time to the first recurrence was $12.1 \pm 6.1$ weeks in the simple aspiration group and $14.7 \pm 6.9$ weeks in the tube thoracostomy group $(p=0.02)$. The rate of recurrence in patients successfully treated with aspiration was 14 out of 48 patients $(29 \%)$, whereas it was six out of 17 patients $(35 \%)$ in whom the aspiration was unsuccessful $(\mathrm{p}=0.3)$.

In the simple aspiration group, 22 patients (34\%) required IM analgesia in the form of pethidine, in the first $24 \mathrm{~h}$ after the procedure versus 40 patients $(56 \%)$ in the tube thoracostomy group $(p=0.01)$. The mean dose of analgesia in the aspiration group was $61.2 \pm 14$ (range: $50-100) \mathrm{mg}$ and $65 \pm 21.7$ (range: $50-125) \mathrm{mg}$ in the tube thoracostomy group $(\mathrm{p}=0.2)$.

TABLE 2 Study primary and secondary end points

\begin{tabular}{|c|c|c|c|c|c|}
\hline \multirow[t]{2}{*}{ Parameters } & \multicolumn{2}{|c|}{ Group } & \multirow{2}{*}{$\begin{array}{c}\text { Difference in proportion } \\
\text { or means }\end{array}$} & \multirow[t]{2}{*}{$95 \% \mathrm{Cl}$} & \multirow[t]{2}{*}{ p-value } \\
\hline & Aspiration $\#$ & Tube thoracostomy & & & \\
\hline Immediate success & $40(62)$ & $49(68)$ & -0.065 & $-0.225-0.094$ & 0.4 \\
\hline 1-week success & $58(89)$ & $63(88)$ & 0.017 & $-0.09-0.124$ & 0.7 \\
\hline Hospitalisation & $17(26)$ & $72(100)$ & -0.738 & $-0.845--0.631$ & $<0.0001$ \\
\hline Hospital stay days & $1.85 \pm 3.9$ & $4 \pm 2.9$ & -2.15 & $-3.3--0.995$ & 0.0003 \\
\hline 3-month recurrence & $10(15)$ & $6(8)$ & 0.071 & $-0.037-0.178$ & 0.2 \\
\hline 1-yr recurrence & $16(25)$ & $17(24)$ & 0.01 & $-0.133-0.153$ & 1 \\
\hline 2-yr recurrence & $20(31)$ & $18(25)$ & 0.057 & $-0.092-0.208$ & 0.5 \\
\hline Time of recurrence weeks & $12.1 \pm 6.1$ & $14.7 \pm 6.9$ & -2.6 & $-4.8--0.389$ & 0.02 \\
\hline Analgesia taken & $22(34)$ & $40(56)$ & -0.217 & $-0.379-0.054$ & 0.01 \\
\hline $\begin{array}{l}\text { Analgesia requirement } \\
\mathrm{mg} \cdot 24 \mathrm{~h}^{-1}\end{array}$ & $61.2 \pm 14$ & $65 \pm 21.7$ & -3.8 & $-10-2.44$ & 0.2 \\
\hline Complications & $1(2)$ & $5(7)$ & -0.054 & $-0.119-0.011$ & 0.2 \\
\hline Inability to work days & $3.6 \pm 0.7$ & $6.04 \pm 1.3$ & -2.44 & $-2.79--2.08$ & $<0.0001$ \\
\hline
\end{tabular}

Data are presented as $n(\%)$ or mean \pm SD. Cl: confidence interval. ${ }^{*}: n=65 ;{ }^{\natural}: n=72$. 


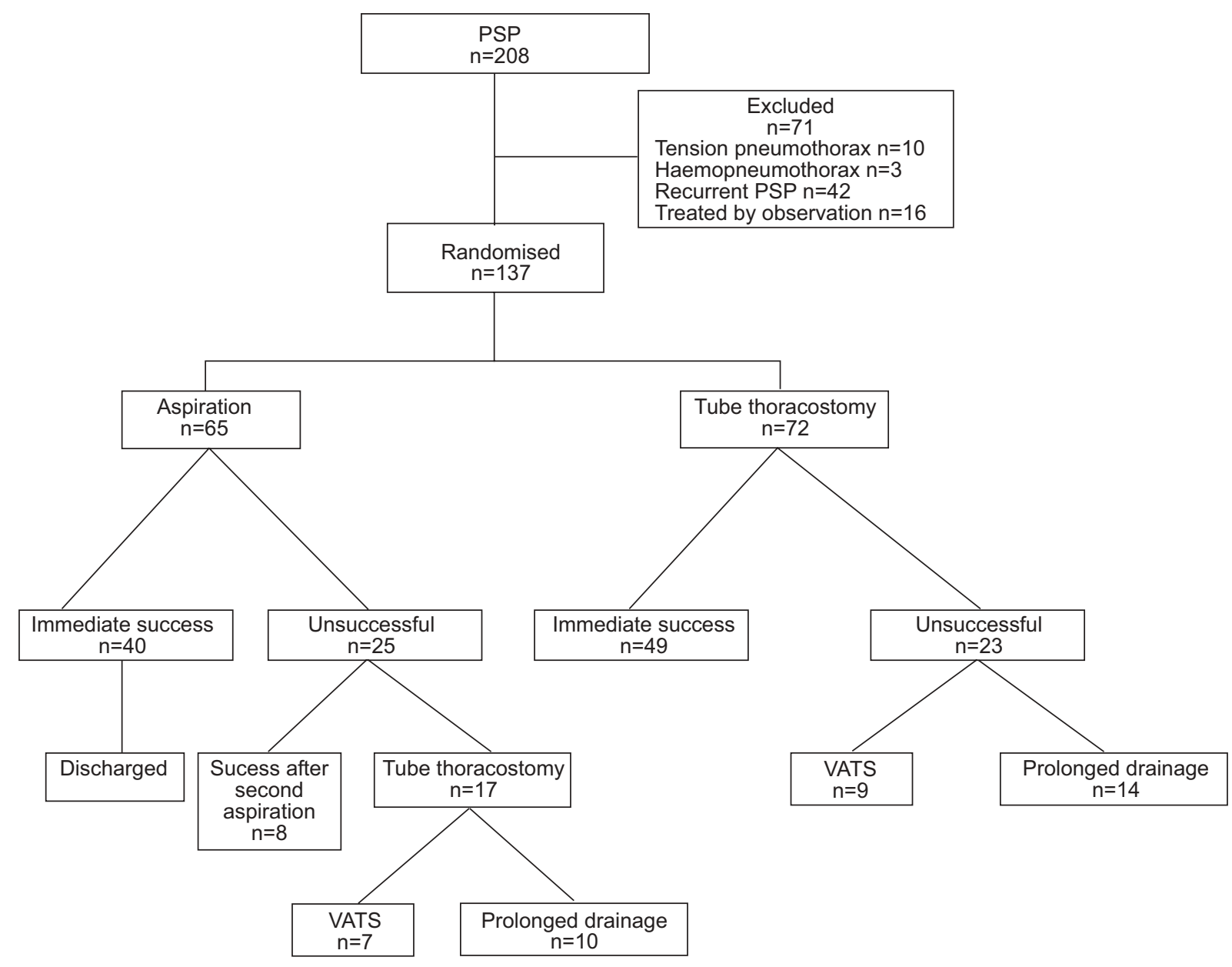

FIGURE 1. Flow diagram of the progress through the treatment allocations and outcome. PSP: primary spontaneous pneumothorax; VATS: video-assisted thoracoscopy surgery.

Complications occurred in six patients $(4 \%)$ and were more common in the tube thoracostomy group (five out of 72 and one out of 65 patients, for the simple aspiration and thoracostomy groups, respectively; $\mathrm{p}=0.2$ ). The complications included subcutaneous emphysema in two patients, tube blockage in a further two patients and exit site infection in one other patient.

The complications in the simple aspiration group only involved one case of subcutaneous emphysema.

\section{DISCUSSION}

In this study, the use of simple aspiration and tube thoracostomy for the first episodes of PSP that cannot be managed by observation were compared and differences in primary and secondary outcomes were determined. The patient population appeared well matched in both groups. The study population represents the target population of PSP, which is predominantly male in the Chest Disease Hospital (Safat, Kuwait). The male predominance is likely to be due to the distribution of smoking patterns. Since very few females smoke in Kuwait [11] and as smoking is a risk factor for pneumothorax, the population distribution was predominantly composed of males. Low incidence of PSP in females may also be a reflection of the generally less active life and average height of females in this part of the world. The results suggest that simple aspiration and tube thoracostomy are equally effective for immediate and long-term success in patients encountering a first episode of PSP. The short-term results also showed that simple aspiration is less painful and associated with fewer complications.

Simple aspiration resulted in a shorter stay in hospital than tube thoracostomy. The attractiveness of simple aspiration is its potential for an outpatient treatment, its relative simplicity, and minimal invasiveness. NOPPEN et al. [8] noted that $>50 \%$ of patients treated by manual aspiration did not require admission and results from the current study confirms the safety of immediate discharge.

There are some issues still to be resolved in the treatment of PSP. These include the efficacy of the two procedures and how well patients can tolerate each procedure. NOPPEN et al. [8] found no significant difference in efficacy between the manual aspiration and chest tube. Moreover, the difference in success rate of simple aspiration compared with chest tube was reported by two other trials, where chest tube drainage achieved a higher success rate $[9,10]$. Therefore, the success rate in studies ranged from $84.8-100 \%$ for chest tubes 
compared with $66.7-92.6 \%$ for simple aspiration [12]. This observation indicates that intercostal tubes allow a rapid and complete re-expansion of the lung and adherence of both visceral and parietal pleura. The present study showed no differences between these two methods.

Recurrence rates at 3 months in the two treatment arms did differ in the present study ( 15 versus $8 \% ; \mathrm{p}=0.2)$. Although this difference is not significant, this observation indicates that the irritating effect of a chest tube on the pleural surfaces may induce aseptic inflammatory response, which helps the pleural surfaces to adhere together and decreases the chances of short-term recurrences of PSP. ANDRIVET et al. [10] reported recurrences of pneumothorax in six out of $29(21 \%)$ and seven out of $24(29 \%)$ patients $(\mathrm{p}>0.45)$, treated by simple aspiration and a chest tube, respectively, in a follow-up examination at 90 days. Similar recurrence rates were found at 12 and 24 months for each method in this series and other studies [8-10]. During a 7-yr study by SCHOENENBERGER et al. [13] recurrence occurred in $34 \%$ of the PSP patients when treated with a chest tube alone. This was similar to a Department of Veterans Affairs cooperative study, by LigHT et al. [14], who found a $32 \%$ recurrence rate in patients treated with chest tube alone. Therefore, despite some discrepancy, these observations indicate that the irritating effect of the chest tube on the pleural surfaces is not sufficient to promote symphysis capable of preventing long-term recurrence.

The current authors observed that an increase in recurrences occurred in patients who had initially been treated unsuccessfully with simple aspiration; 14 out of 48 patients $(29 \%)$ in the successful group compared with 6 out of 17 patients (35\%) in those where aspiration was unsuccessful. Although this difference is not significant, patients in whom aspiration is successful may have smaller blebs than those in whom it fails. The other explanation is the efficiency of pleural opposition. Simple aspiration may not be effective if the parenchymal tear is large enough and theoretically carries a high risk of shortterm recurrence, because it does not promote pleural symphysis. SCHRAMEL et al. [2] reported that the presence of emphysema like changes (ELCs) in patients with PSP had no predictive value for the future development of recurrences and, therefore, the choice of treatment should not depend on the presence of ELCs but on the efficacy of the treatment.

Chest tube insertion is associated with higher levels of pain and anxiety [15], and opioid premedication and local anaesthesia is required. HARVEY et al. [9] reported lower total pain scores in the simple aspiration group compared with the chest tube group. Similarly, in the current study, analgesia was required in 40 out 72 patients $(56 \%)$ in the tube thoracostomy group and 22 out of 65 patients (34\%) in the simple aspiration group $(p=0.01)$. However, the mean amount of analgesia was not statistically significant between the two groups. ANDRIVET et al. [10] reported that patients with a spontaneous pneumothorax, who were left untreated, experienced as much pain as those who had undergone an insertion of a chest tube.

The primary limitation of this trial was that it was underpowered to definitively demonstrate equality of the two treatment arms. Previous studies also had the same limitation.
Clinical application and interpretation of this study should take this limitation into account. However, this study does suggest, with $95 \%$ confidence intervals, that the use of a chest tube is not likely to be $>9.4 \%$ benefit over simple aspiration in the immediate success of the procedure (the primary outcome). Larger trials may be needed to answer the treatment question definitively. A second limitation involved the inability to mask the patients or investigators to the treatment arm. Lack of masking can lead to information bias. However, the investigators used standard clinical definitions of pneumothorax resolution in evaluating "success" of resolution or recurrence of pneumothorax. It is unlikely, therefore, that any significant bias was present in the outcome ascertainment.

In conclusion, the current study's results demonstrate the safety and efficacy of simple aspiration in the treatment of first episodes of primary spontaneous pneumothorax that cannot be managed by observation. The advantage of this option is its availability, low procedure-related morbidity, reduced pain, and lower hospitalisation rate. Therefore, the current authors recommend simple aspiration as an alternative treatment option to tube thoracostomy in the initial treatment of a first episode of primary spontaneous pneumothorax.

\section{REFERENCES}

1 Gupta D, Hansell A, Nichols T, Duong T, Ayres JG, Strachen S. Epidemiology of pneumothorax in England Thorax 2000; 55: 666-671.

2 Schramel FMNH, Postmus PE, Vanderschueren RGJRA. Current aspects of spontaneous pneumothorax. Eur Respir J 1997; 10: 1372-1379.

3 Baumann MH, Strange C. Treatment of spontaneous pneumothorax. A more aggressive approach. Chest 1997; 112: 789-804.

4 Baumann $\mathrm{MH}$, Noppen M. Pneumothorax. Respirology 2004; 9: 157-164.

5 Baumann MH, Strange C, Heffner JE, et al. Management of spontaneous pneumothorax: an American College of Chest Physicians Delphi consensus statement. Chest 2001; 119: 590-602.

6 Miller AC, Harvey JE. Guidelines for the management of spontaneous pneumothorax. BMJ 1993; 307: 114-116.

7 Henry H, Arnold T, Harvey J. BTS guidelines for the management of spontaneous pneumothorax. Thorax 2003; 58: Suppl. 2, ii39-ii58.

8 Noppen M, Alexander P, Driesen P, Slabbynck H, Verstraeten A. Manual aspiration versus chest tube drainage in first episodes of primary spontaneous pneumothorax. Am J Respir Crit Care Med 2002; 165: 1240-1244.

9 Harvey J, Prescott RJ. Simple aspiration versus intercostal tube drainage for spontaneous pneumothorax in patients with normal lungs. BMJ 1994; 309: 1338-1339.

10 Andrivet P, Djedaini K, Teboul JL, Brochard L, Dreyfuss D. Spontaneous pneumothorax: comparison of thoracic drainage vs. immediate or delayed needle aspiration. Chest 1995; 108: 335-339.

11 Moody PM, Memon A, Sugsthsn TN, El-Gerges NS, Al-Bostan M. Factors associated with the initiation of smoking by Kuwaiti males. J Subst Abuse 1998; 10: 375-384. 
12 Devanand A, Koh MS, Ong TH, et al. Simple aspiration versus chest-tube insertion in the management of primary spontaneous pneumothorax: a systematic review. Respir Med 2004; 98: 579-590.

13 Schoenenberger RA, Haefeli WE, Weiss P, Rirz RF. Timing of invasive procedures in therapy for primary and secondary spontaneous pneumothorax. Arch Surg 1991; 126: 764-766.
14 Light RW, O'Hara VS, Moritz TE, et al. Intrapleural tetracycline for the prevention of recurrent spontaneous pneumothorax: results of a Department of Veterans Affairs cooperative study. JAMA 1990; 264: 2224-2230.

15 Luketich JD, Kiss MD, Hershey J, et al. Chest tube insertion: a prospective evaluation of pain management. Clin J Pain 1998; 14: 152-154. 